Convergence of a Two-Stage Richardson

Iterative Procedure for

Solving systems of Linear Equations ${ }^{\ddagger}$

Gene H. Golub* and Michael I. Overtone

September 1981

REPORT NO. 38

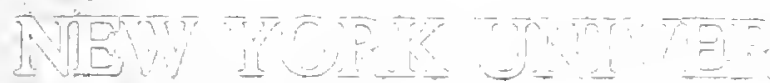

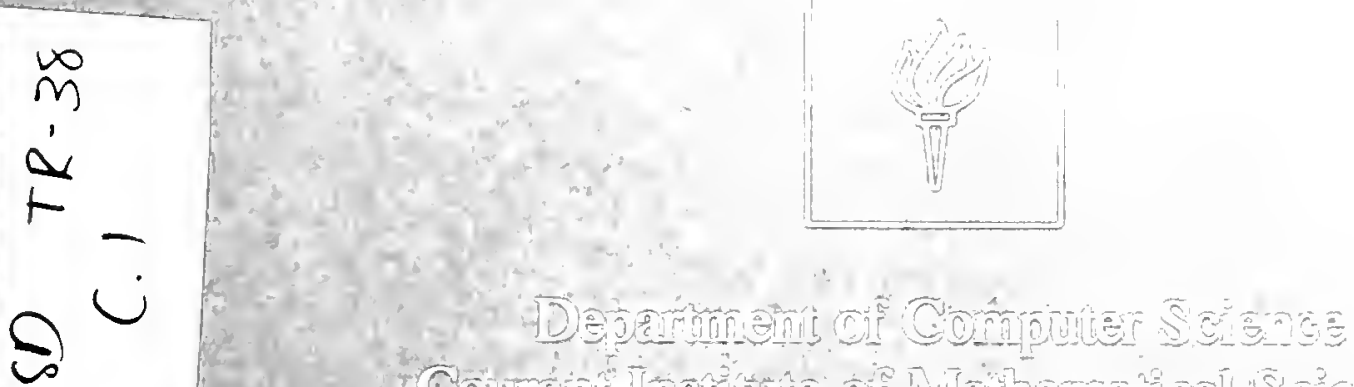

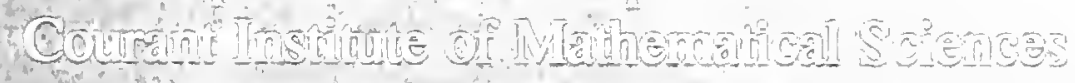

251 N 


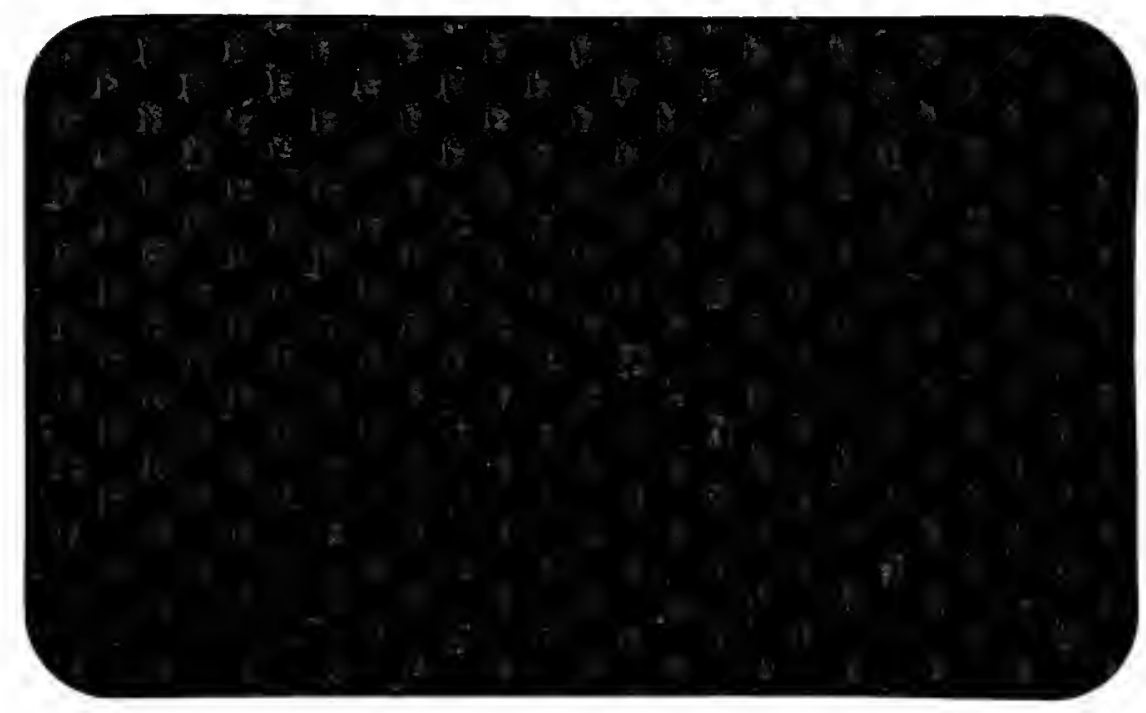




\section{Convergence of a Two-Stage Richardson Iterative Procedure for Solving systems of Linear iquations ${ }^{\ddagger}$ Gene H. Golub* and Michael L. Overton ${ }^{\dagger}$ September 1981}

REPORT NO. 38

$\neq$

Presented at the Dundee Biennial Conference on Numerical Analysis, University of Dundee, Scotland, June 23-26, 1981. To appear in the Proceedings of the conference, published by springer-Verlag.

* Computer science Department, stanford University, stanford, California 94305, U.S.A. Supported in part by the United States Department of Energy contract DE-AT03-ER71030 and in part by the National Science Foundation grant MCS-78-11985.

† Computer Science Department, Courant Institute of Mathematical Sciences, New York University, 251 Mercer st., New York, NY 10012, U.S.A. Supported in part by the United States Department of Energy contract DE-AC02-76ER03077 and in part by the National Science Foundation grant MCS-81-01924. 
$\therefore$ 
Gene H. Golub* and Michael L. Overton ${ }^{*}$

0 . Introduction

Consider the problem of solving a system of linear equations

$$
A x=b
$$

by an iterative method, i.e. generating a sequence of approximations $\left\{x_{k}\right\}$ such that $\lim _{k \rightarrow \infty} x_{k}=x$. Frequently it is useful to introduce a splitting

$$
A=M-N
$$

where systems of the form

$$
M Y=c
$$

may be solved much more easily than the original system (0.1). When (0.1) arises from the discretization of a partial differential equation, such a splitting is often natural, with $M$ and $N$ corresponding to separate terms in the differential equation. The iterative method used to solve (0.1) can then be "preconditioned" by M, i.e. designed so that each step of this "outer" iteration involves solving a system of the form (0.2). Sometimes it is desirable to solve these systems (0.2) by "inner" iterative procedures. In this paper we consider using the second-order Richardson method for the outer iteration, and show how the rate of convergence of this iteration depends on the accuracy re-

\footnotetext{
Computer science Department, Stanford University, Stanford, California 94305, U.S.A. Supported in part by the United States Department of Energy contract DE-AT03-ER71030 and in part by the National science Foundation grant MCS-78-11985.

Computer Science Department, Courant Institute of Mathematical Sciences New York University, 251 Mercer St., New York, NY 10012, U.S.A. Supported in part by the United States Department of Energy contract DEAC02-76ER03077 and in part by the National science Foundation grant MCS-81-01924.
}

$+$ 
quirec for the inner iterations. To our knowledge analysis of this particular method has not been attempted previously. See Nichols (1973) for an analysis of more general schemes using inner and outer iterations to solve linear systems. Other related papers on the solution of linear or nonlinear problems by two-stage methods include Gunn (1964), Nicolaides (1975), Pereyra (1967) and Dembo, Eisenstat and Steihang (1980). See Golub (1962) Eor a stuay of round-off error for the Richarcson method, which we do not explicitly consider here.

One motivation for our work is that the two-stage method can be effectively used to solve nonsymetric systems where $M$ is symmetric positive definite and $N$ is skew-symmetric. In this case the symmetric/ skew-symmetric splitting $Z=M-N$ is used to precondition the outer iteration, and a symmetric splitting $M_{i}=M_{1}-M_{2}$ can be used to precondition each inner iteration, using a direct method to solve a system of the form $M_{1} Y=c$ at each step of each irner iteration. (See Manteuffel (1977) Eor alternative approaches for nonsymetric systems.) Numerical results from applying the Richardson method to such nonsymmetric systems are given in section 3. We also present numerical results using the conjugate gradient method for the outer iteration. Analysis of the latter procedure would be very interesting, but this seems more difizcult.

We use \|\| to cenote the Euclidean vector and matrix norm, defined by

$$
\|x\|=\left(x^{T} x\right)^{1 / 2},\|B\|=\max _{\|x\|=0} \frac{\|B x\|}{\|x\|} .
$$

1. Symmetric Dositive definite systems.

Let us First assume that both $A$ and $M$ are symmetric and positive definite. Consider the following iterative method.

\section{Method I.}

Choose positive scalar parameters $\delta, \alpha$ and $\omega$, with $0 \leq \delta<1$. Choose initial vectors $x_{0}$ and $x_{1}$. For $k=1,2, \ldots$, define

$$
x_{k+1}=x_{k-1}+\omega\left(\alpha z_{k}+x_{k}-x_{k-1}\right)
$$

where

$$
\begin{aligned}
M z_{k} & =r_{k}+q_{k}, \\
r_{k} & =b-A x_{k},
\end{aligned}
$$


and

$$
\left\|q_{k}\right\| \leq \hat{o}\left\|r_{k}\right\|
$$

If $\hat{c}=0$ then Method 1 reduces to the second-order Richardson method. The conditions on the linear operator and on the parameters $\alpha$ and w under which convergence is guaranteed are well known in this case (see Golub and Varga (1961)). When $\delta>0$ the meaning of (1.2) and (1.3) is that the system

$$
M z=r_{k}
$$

is being "solved" by an inner iterative procedure, which is terminated when the residual norm $\left\|_{k}\right\|$ has been sufficiently reduced. If the outer (main) iteration is converging to the solution of (0.1), the associated residuals $\left\{r_{k}\right\}$ are converging to zero, and hence $z=0$ is a reasonable starting point for each inner iteration. Equation (1.3) then specifies that each inner iteration must reduce its associated residual norm by a factor of $\delta$. Note that the early inner iterations solve (1.4) with low absolute accuracy, while the later systems are solved with high accuracy. There is no restriction on the type of method used for the inner iterations.

\subsection{Convergence analysis}

Let us now analyse the convergence of Method 1 . Let the error at each step of the outer iteration be

$$
e_{k}=x-x_{k}
$$

where $x$ is the solution of $(0.1)$. Note that $r_{k}=A e_{k}$ for all $k$. For $k=1,2, \ldots$, we have:

$$
\begin{aligned}
e_{k+1} & =e_{k-1}-\omega\left(\alpha z_{k}+e_{k-1}-e_{k}\right) \\
& =\omega K e_{k}+(1-\omega) e_{k-1}+p_{k}
\end{aligned}
$$

where

$$
p_{k}=-w \alpha M^{-1} q_{k}
$$

and

$$
K=I-\alpha M^{-1} A .
$$


Now $K$ is similar to a symmetric matrix, and we can define its eigenvector decomposition by

$$
\mathrm{K}=\mathrm{M}^{-1 / 2}\left(I-\alpha \mathrm{M}^{-1 / 2} \mathrm{AM}^{-1 / 2}\right) \mathrm{M}^{1 / 2}=\mathrm{M}^{-1 / 2} \mathrm{~V} \Sigma \mathrm{V}^{\mathrm{T}} \mathrm{M}^{I / 2}
$$

where $\sum$ is a diagonal matrix of eigenvalues $\left\{\sigma_{j}\right\}, j=1, \ldots, n$, and $V$ is orthogonal. Let

$$
\hat{e}_{k}=V^{T} M^{1 / 2} e_{k} \text { and } \hat{p}_{k}=V^{T} M^{1 / 2} p_{k}=-\omega \alpha V^{T} M^{-1 / 2} q_{k} .
$$

Then from (1.5) we obtain the diagonalized system of difference equations:

$$
\hat{e}_{k+1}=\omega \Sigma \hat{e}_{k}+(1-\omega) \hat{e}_{k-1}+\hat{p}_{k}, k=1,2, \ldots .
$$

At this point we state a lemma which can be proved using the standard theory of difference equations.

Lemma 1. Consider the inhomogeneous difference equation in $\xi_{k}$ :

$$
\xi_{k+1}=\beta \xi_{k}+\gamma \xi_{k-1}+n_{k, k}=1,2, \ldots .
$$

The solution to $(1.10)$ is given by

$$
\xi_{k}=\theta_{k} \xi_{1}+\gamma \theta_{k-1} \xi_{0}+\sum_{\ell=1}^{k-1} \theta_{k-l} \eta_{l}
$$

where

$$
\theta_{k}= \begin{cases}\frac{\lambda_{1}^{k}-\lambda_{2}^{k}}{\lambda_{1}-\lambda_{2}} & \text { if } \lambda_{1} \neq \lambda_{2} \\ k \lambda_{1}^{k-1} & \text { if } \lambda_{1}=\lambda_{2}\end{cases}
$$

and where $\lambda_{1}$ and $\lambda_{2}$ are the roots of the characteristic polynomical

$$
\lambda^{2}-\beta \lambda-\gamma=0
$$

(Note that if the coefficients of (1.10) are real, $\lambda_{1}$ and $\lambda_{2}$ may be complex but $\theta_{k}$ is real).

It follows from Lemma 1 that the solution to (1.7) is

$$
\hat{e}_{k}=s_{k} \hat{e}_{1}+(1-\omega) s_{k-1} \hat{e}_{0}+\sum_{\ell=1}^{k-1} s_{k-\ell} \hat{p}_{\ell}, k=1,2, \ldots
$$


where $S_{k}$ is a diagonal matrix with $j^{\text {th }}$ diagonal element

$$
\left(s_{k}\right)_{j j}= \begin{cases}\frac{\lambda_{1, j}-\lambda_{2, j}}{\lambda_{1, j}-\lambda_{2, j}} & \text { if } \lambda_{1, j} \neq \lambda_{2, j} \\ k \lambda_{1, j} k-1 & \text { if } \lambda_{1, j}=\lambda_{2, j}\end{cases}
$$

and where $\lambda_{1, j}$ and $\lambda_{2, j}$ are the roots of

$$
\lambda^{2}-\omega \sigma_{j} \lambda+(\omega-1)=0, \quad j=1, \ldots, n .
$$

Now let

$$
\rho=\max _{1 \leq j \leq n}\left(\max \left(\left|\lambda_{1, j}\right|,\left|\lambda_{2}, j\right|\right)\right)
$$

It can easily be shown (by induction on $k$ ) that

$$
\left\|S_{k}\right\| \max _{1 \leq j \leq n}\left|\left(S_{k}\right)_{j j}\right| \leq k p^{k-1} .
$$

Note also from (1.13) that the product of the roots $\lambda_{1, j} \lambda_{2, j}$ is equal to $w-1$, so $0^{2} \geq|\omega-1|$. We therefore have from (1.11) that

$$
\left\|\hat{e}_{k}\right\| \leq k \rho^{k-1}\left\|\hat{e}_{1}\right\|+(k-1) \rho^{k}\left\|\hat{e}_{0}\right\|+\sum_{\ell=1}^{k-1}(k-\ell) \rho^{k-\ell-1}\left\|\hat{p}_{\ell}\right\|, k=1,2, \ldots
$$

At this point we need a lemma relating $\left\|\hat{p}_{k}\right\|$ to $\left\|\hat{e}_{k}\right\|$.

Lemma 2. Assume that (1.3) holds. Then

$$
\left\|\hat{p}_{k}\right\| \leq \varepsilon\left\|\hat{e}_{k}\right\|, \quad k=1,2, \ldots
$$

where $\varepsilon=\delta \omega \alpha\left\|M^{-1 / 2}\right\|\left\|A M^{-1 / 2}\right\|$.

Proof. By (1.8) and (1.3) we have:

$$
\begin{aligned}
\left\|\hat{p}_{k}\right\| & \leq \omega \alpha\left\|M^{-1 / 2}\right\|\left\|q_{k}\right\| \\
& \leq \delta \omega \alpha\left\|M^{-1 / 2}\right\|\left\|r_{k}\right\|
\end{aligned}
$$




$$
\leq \delta \omega \alpha\left\|M^{-1 / 2}\right\|\left\|A M^{-1 / 2} V^{T} M^{1 / 2} e_{k}\right\|
$$

from which the result follows.

Continuing with the error analysis, we can substitute $\varepsilon\left\|\hat{e}_{l}\right\|$ for $\left\|\hat{p}_{\ell}\right\|$ in (1.15), and rewrite it to obtain

$$
\begin{array}{r}
\left\|\hat{e}_{k}\right\|-\varepsilon \sum_{\ell=1}^{k-1}(k-\ell) 0^{k-\ell-1}\left\|\hat{e}_{\ell}\right\| \leq k 0^{k-1}\left\|\hat{e}_{1}\right\|+(k-1) \rho^{k}\left\|\hat{e}_{0}\right\|, \\
k=1,2, \ldots, m,
\end{array}
$$

where $\mathrm{m}$ is introduced to indicate the last computed iterate $\mathrm{x}_{\mathrm{m}}$. This system of $m$ linear inequalities can be written using matrix notation as

$$
(I-\varepsilon L)\left[\begin{array}{c}
\left\|\hat{e}_{I}\right\| \\
\left\|\hat{e}_{m}\right\|
\end{array}\right] \leq s
$$

where the non-negative strictly lower triangular matrix I and the vector s are defined accordingly. Now $L^{m}=0$ so

$$
(I-\varepsilon I)^{-1}=I+\varepsilon I+\varepsilon^{2} I^{2}+\ldots+\varepsilon^{m-1} L^{m-1} .
$$

Thus $(I-\varepsilon L)^{-1}$. is also a non-negative matrix and hence

$$
\left[\begin{array}{c}
\left\|\hat{e}_{1}\right\| \\
\left\|\hat{e}_{m}\right\|
\end{array}\right] \leq(I-\varepsilon L)^{-1} s .
$$

Let us define $t=\left[\tau_{1}, \ldots, \tau_{m}\right]^{T}=(I-\varepsilon I)^{-1} s$. By definition, $\tau_{k}$ satisfies

$$
\begin{array}{r}
\tau_{k}=k \rho^{k-1}\left\|\hat{e}_{1}\right\|+(k-1) \rho^{k} \hat{e}_{0} \|+\varepsilon \sum_{\ell=1}^{k-1}(k-\ell) \rho^{k-\ell-1} \tau_{\ell} \\
k=1,2, \ldots, m .
\end{array}
$$

The first equation defines $\tau_{1}=\left\|\hat{e}_{l}\right\|$. For convenience, we now define $\tau_{0}=-\left\|\hat{e}_{0}\right\|$, and it then follows from Lemma $l$ that $\tau_{k}$ is the solution to a difference equation of the form (1.10) with inhomogeneous term $\varepsilon \tau_{k}$ and characteristic polynomial with a double root, i.e. 


$$
\tau_{k+1}=2 \rho \tau_{k}-\rho^{2} \tau_{k-1}+\varepsilon \tau_{k}, \quad k=1,2, \ldots .
$$

This, however, can be viewed as the homogeneous equation

$$
\tau_{k+1}=(2 \rho+\varepsilon) \tau_{k}-\rho^{2} \tau_{k-1}
$$

and hence, using Lemma I again, has solution

$$
\tau_{k}=\frac{v_{1}^{k}-v_{2}^{k}}{v_{1}-v_{2}} \tau_{1}-\rho^{2} \frac{v_{1}^{k-1}-v_{2}^{k-1}}{v_{1}-v_{2}} \tau_{0},
$$

where $v_{1}$ and $v_{2}$ are the roots of

$$
\lambda^{2}-(2 \rho+\varepsilon) \lambda+\rho^{2}=0 \text {. }
$$

If we define $\tilde{\rho}=\max \left(v_{1}, v_{2}\right)$ we have

$$
\begin{aligned}
\tilde{\rho} & =\rho+\frac{\varepsilon}{2}+\left(\rho \varepsilon+\frac{\varepsilon^{2}}{4}\right)^{1 / 2} \\
& =\rho+\rho^{1 / 2} \varepsilon^{1 / 2}+\frac{\varepsilon}{2}+0\left(\varepsilon^{3 / 2}\right) \text {, if } \varepsilon \ll 1 .
\end{aligned}
$$

Using (1.18) and (1.19) we now have a condition which guarantees the convergence of the error norms $\left\{\left\|\hat{e}_{k}\right\|\right\}$ to zero, namely $\tilde{p}<1$. More specifically, we have the following result:

Theorem 1. The error norm $\left\|\hat{e}_{k}\right\|$ associated with the $k^{\text {th }}$ iterate of Method 1 is bounded by

$$
\left\|\hat{e}_{k}\right\| \leq k \tilde{\rho}^{k-1}\left\|\hat{e}_{1}\right\|+(k-1) \tilde{\rho}^{k}\left\|\hat{e}_{0}\right\|,
$$

where $\tilde{\rho}=\tilde{\rho}(\delta, \alpha, \omega)$ is given by $(1.20),(1.16),(1.14),(1.13)$ and $(1.7) \cdot \square$ Note that if $\delta=\varepsilon=0$, we have $\tilde{\rho}=\rho$ and Theorem 1 reduces to the standard convergence property of the second-order Richardson method (see Golub and Varga (1961)). We also note that it is possible to choose the first iterate $x_{1}$ in such a way that the error bound of Theorem 1 is somewhat reduced. 


\subsection{Optimal parameters.}

There are three Eree parameters in Method 1, namely $\delta$, a and $\omega$. Let us first suppose that $\delta=0$, so that the inner systems (1.4) are solved exactly. In this case the optimal choices of $\alpha$ and w, i.e. those that minimize $p$ in (1.14), may be described using young's theory of successive overrelaxation. Let

$$
\left|\sigma_{l}\right|=\max _{1 \leq j \leq n}\left|\sigma_{j}\right|
$$

where $\left\{\sigma_{j}\right\}$ are the eigenvalues of $K=I-\alpha M^{-1} A$, as before. Given any $\alpha$, the optimal choice of $\omega$ is obtained by choosing $\lambda_{1, l}=\lambda_{2, l}=\frac{1}{2} \omega \sigma_{l}$ in $(1.13), i . e$.

$$
\omega=\frac{2}{1+\sqrt{1-\sigma_{l}^{2}}} .
$$

It can be verified that this choice results in

$$
\rho=\left|\lambda_{I, \ell}\right|=\left|\lambda_{I, j}\right|=\left|\lambda_{2, j}\right|=\frac{I}{2} \omega\left|\sigma_{\ell}\right|=\sqrt{\omega-I}
$$

for all $j, j=1, \ldots, n$. Note that $(1.21)$ implies $1 \leq w<2$. See Varga (1962,p.109) or Young (1971,p.171) for the proof that (1.21) is optimal, and see Golub and Varga (196I) for a description of the connection between the Richardson and successive overrelaxation methods. Since $\rho=\frac{1}{2} \omega\left|\sigma_{l}\right|$, and $\omega$ satisfies (1.21), it is clear that $\alpha$ should be chosen to minimize $\left|\sigma_{l}\right|$, the spectral radius of $k$. Let $u_{\max }$ and $\mu_{\min }$ be respectively the maximum and minimum eigenvalues of $M^{-1} A$. Clearly $\left|\sigma_{l}\right|$ is minimized by specifying

$$
1-\alpha \mu_{\min }=-\left(1-\alpha \mu_{\max }\right),
$$

i.e.

$$
\alpha=\frac{2}{\mu_{\max }+\mu_{\min }}
$$

and

$$
\left|\sigma_{\ell}\right|=\frac{\mu_{\max }-\mu_{\min }}{\mu_{\max }+\mu_{\min }}<1 .
$$

When we permit $\delta>0$ the optimal choice of parameters is more complicated. Our aim is to minimize the amount of work needed to obtain 
a solution of prescribed accuracy. A reasonable measure of the total amount of work required for generating $x_{1}, \ldots, x_{m}$ is

$$
w=\sum_{k=1}^{m} m_{k}
$$

where $m_{k}$ is the number of iterates required in the $k^{\text {th }}$ inner iteration to achieve (1.2) and (1.3). This measure is particularly appropriate when each step of every inner iteration involves solving another system of equations by a direct method. Now let $\gamma$ be a measure of relative accuracy prescribed for the outer iteration, e.g. specifying $\left\|\hat{e}_{m}\right\| \leq$ $\gamma\left\|\hat{e}_{0}\right\|$. Roughly speaking, Theorem I says that the error is reduced by approximately a factor of $\tilde{p}$ at each step of the outer iteration (this neglects $\left\|\hat{e}_{I}\right\|$ and a factor of $\left.(k+l) / k\right)$. With this assumption the number of iterates required for the outer iteration is approximately

$$
m \approx \frac{-\log \gamma}{-\log \hat{\rho}(\delta, \alpha, \omega)}
$$

Now let us suppose that the iterative method used for the inner iteration is such that the associated residual is reduced by about a factor of $\theta$ at each step. Assuming a starting point $z=0$ for each inner iteration we have

$$
\mathrm{m}_{\mathrm{k}} \approx \frac{-\log \hat{\delta}}{\log \theta} \quad, \mathrm{k}=1, \ldots, \mathrm{m}
$$

Under these assumptions the total amount of work $W$ is about

$$
W \approx \frac{-\log \gamma}{-\log \theta} \cdot \frac{-\log \delta}{-\log \rho(\delta, \alpha, \omega)}
$$

Now $Y$ and $\theta$ are fixed so a reasonable goal in choosing the parameters $\delta, \alpha$ and $\omega$ is to minimize

$$
\bar{W}=\frac{-\log \delta}{-\log \tilde{\rho}(\delta, \alpha, \omega)}
$$

Naturally this is difficult to do, but the formula gives some insight. As $\delta \rightarrow 0, \bar{W} \rightarrow \infty$, indicating that solving the inner iterations too accurately is very expensive. On the other hand, if $\delta$ is allowed large enough so that $\tilde{p} \rightarrow 1$, we have $\bar{W} \rightarrow \infty$, indicating that the outer iteration is not convergent. The optimal value of $\delta$, given $\alpha$ and $w$, is somewhere in between these extremes.

The optimal values of $\alpha$ and $w$ are equally complicated, since $\tilde{\rho}$ de- 
pends on them through both $p$ and $\varepsilon$, but it seems likely that (1.21) and (1.22) would be good choices. One reasonable way to choose the parameters in practice is to make several numerical experiments. FortunateIy, once an adequate choice of parameters is determined for a particular problem, whole classes of related problems can usually be solved efficiently with the same parameter values. Another interesting and promising idea is to try to choose $\delta$ dynamically, as the outer iteration. proceeds.

\section{Symmetric/skew-symmetric splittings.}

Method 1 can be used to solve (0.1) if $A$ is nonsymmetric but with a positive definite symmetric part. We define the splitting $A=M-N$ by

$$
M=\frac{1}{2}\left(A^{T}+A\right), N=\frac{1}{2}\left(A^{T}-A\right)
$$

where $M$ and $N$ are respectively called the symmetric and skew-symmetric parts of $A$, and $M$ is positive definite. The matrix $K$ is given by

$$
K=I-\alpha M^{-1} A=(I-\alpha) I+\alpha M^{-1} N
$$

Now $M^{-1} N$ is similar to the skew-symmetric matrix $M^{-1 / 2} \mathrm{NM}^{-1 / 2}$, and thus its eigenvalues, say $\left\{i k_{j}\right\}, j=1, \ldots, n$, are imaginary. The eigenvalues of $k$ are thus given by

$$
\sigma_{j}=(1-\alpha)+i \alpha k_{j}
$$

Let us now investigate the choice of optimal parameters when $\delta=0$. Young (1971,p.196) gives formulae for the optimal choice of $w$ and the resulting value of $p$ in the case when the eigenvalues of $k$ are complex but confined to the rectangle defined by the real and imaginary parts of the largest eigenvalue in modulus, a property which (2.1) satisfies. It can be verified that $p$ is minimized when $\alpha$ in (2.1) is chosen by

$$
\alpha=1 \text {. }
$$

With this choice the eigenvalues $\left\{\sigma_{j}\right\}$ are imaginary, and the optimal choice of $w$ reduces to $(1.21)$. Notice that now $\lambda_{1, l}=\lambda_{2, l}$ is imaginary and $0<\omega \leq 1$. 
Having motivated the choice (2.2), we now permit $\delta>0$ and consider the convergence of Method l using the symmetric/skew-symmetric splitting, with $\alpha=1$. All of the error analysis, including Theorem 1 , applies to this case, provided only that we replace $\mathrm{V}^{\mathrm{T}}$ by $\mathrm{V}^{\mathrm{H}}$ in (1.7), where $V$ is now a complex unitary matrix (and $\Sigma$ is imaginary).

\section{Numerical Experiments}

Consider the differential equation:

$$
-\Delta u+(a u)_{x}+a u_{x}+(b u)_{y}+b u_{y}+c \bar{u}=f
$$

where $u, a, b$ and $f$ are functions defined on the unit square: $0 \leq x \leq 1$, $0 \leq y \leq 1$. Approximating (3.1) by second-order finite differences on a grid with $t$ interior points in each direction results in an $t^{2} \times t^{2}$ linear system to be solved:

$$
(M-N) u_{t}=d
$$

Here $u_{t}$ is the solution to the discretized problem, $N=-N^{T}$ is a skewsymmetric matrix corresponding to the first-order terms in (3.1), $M=M^{T}=M_{1}-M_{2}$ is a symmetric matrix, with $M_{1}$ the negative discrete Laplacian operator and $\mathrm{M}_{2}$ a diagonal matrix corresponding to the zeroorder term, and $d$ corresponds to $f$, modified to account for the boundary conditions. We consider the particular differential equation whose solution is $u(x, y)=\exp \left(x^{2}+y^{2}\right)$, with coefficients $a(x, y)=b(x, y)=$ $5 \exp \left(x^{2}+y^{2}\right), c(x, y)=10 \exp \left(3 x^{2}+3 y^{2}\right)$, and with nonhomogeneous Dirichlet conditions imposed on the boundary of the square.

Table I gives the result of solving (3.2) by Method 1 . Note that the symmetric/skew-symmetric splitting was used for the outer iteration, as discussed in section 2. The parameters were $\alpha=1$ and w given by (1.21) (see below). The initial vector $x_{0}$ was set to zero, and $x_{1}$ was obtained by: $x_{1}=z_{0}+x_{0} ; M_{0}=r_{0}+q_{0} ;\left\|q_{0}\right\| \leq \delta\left\|r_{0}\right\|$. The termination criterion was $\left\|r_{m}\right\| \leq 10^{-8}$. Each inner iteration used a (preconditioned) conjugate gradient method, starting with the zero vector, to approximately solve a system of the form $M z=r$, in the sense of (1.2) and (1.3). The symmetric splitting $M=M_{1}-M_{2}$ was exploited so that each step of the inner iteration used a fast direct method to solve a system of the form $M_{I} \bar{z}=\bar{r}$, i.e. the Poisson equation.

In addition to the Richardson results, Table 1 also gives the 
results of experiments using a (preconditioned) conjugate gradient method for the outer iteration to solve (3.2). See concus and Golub (1976) and widlund (1978) for a discussion of this method, which exploits the symmetric/skew-symmetric splitting. The inner iterations were carried out exactly as above, being terminated by (1.2) and (1.3). The conjugate gradient method was also run with the inner iterations carried out to machine precision, in order to obtain $\sigma_{l}$ from the resulting tridiagonal Lanczos matrix. These runs, made solely to obtain the "optimal" w defined by (1.2l), are not included in the table.

\section{Table 1.}

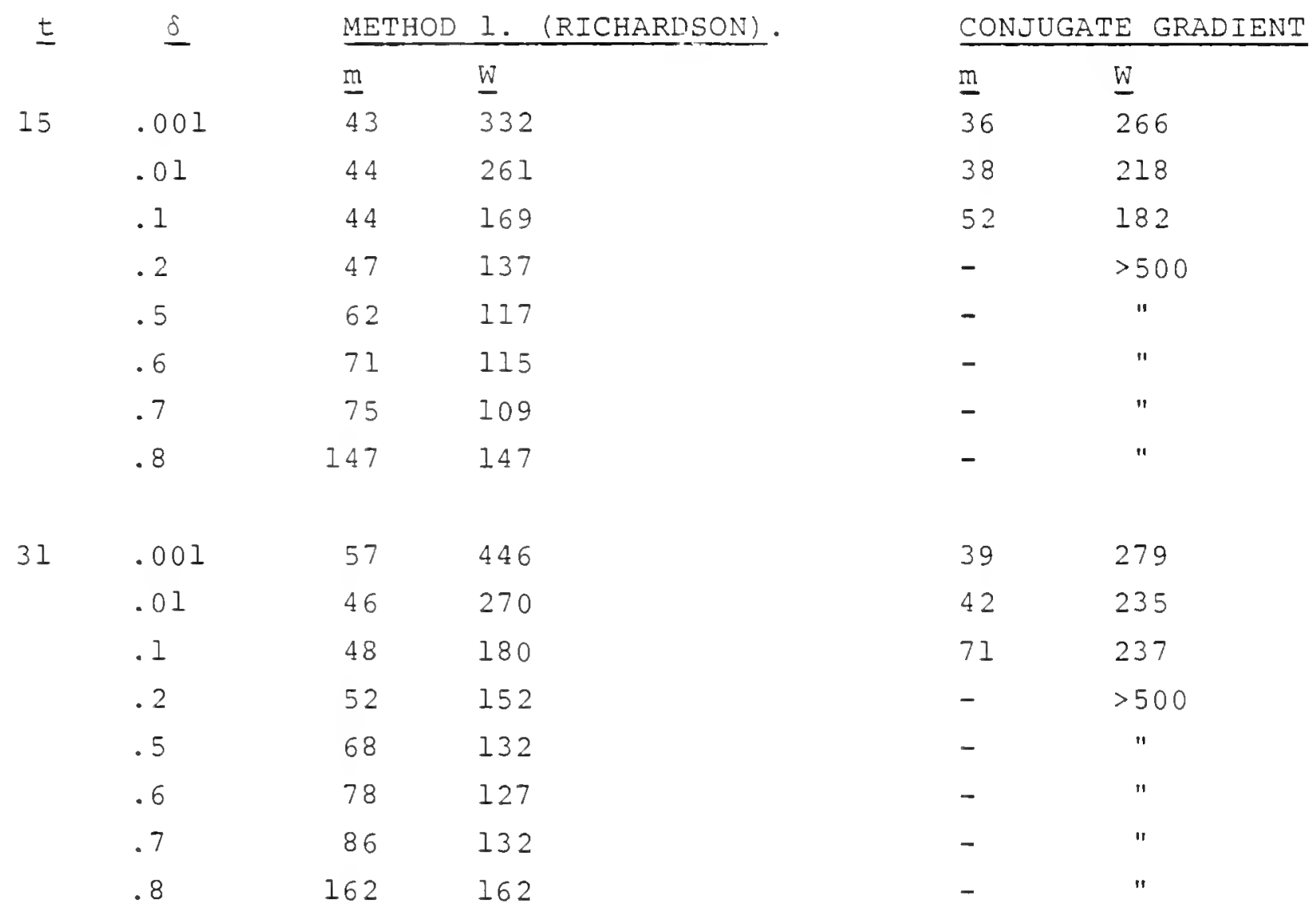

Note: $t$ is the number of mesh points in each direction, $\delta$ defines (1.4), $m$ is the number of outer iterates and $W$ is the total number of inner iterates (number of calls to the fast Poisson solver).

The results of Table 1 are graphed in Figure 1 . Notice that the optimal choice of $\delta$, given $\omega$ and $\alpha$, is quite well-determined by the curves, and has a value which can be close to one. Using a near optimal value of $\delta$ is far more efficient than carrying out the inner iterations to full accuracy. It is interesting to note that the Richardson method (with near optimal $\omega$, in practice unknown) is less efficient than the 
\&

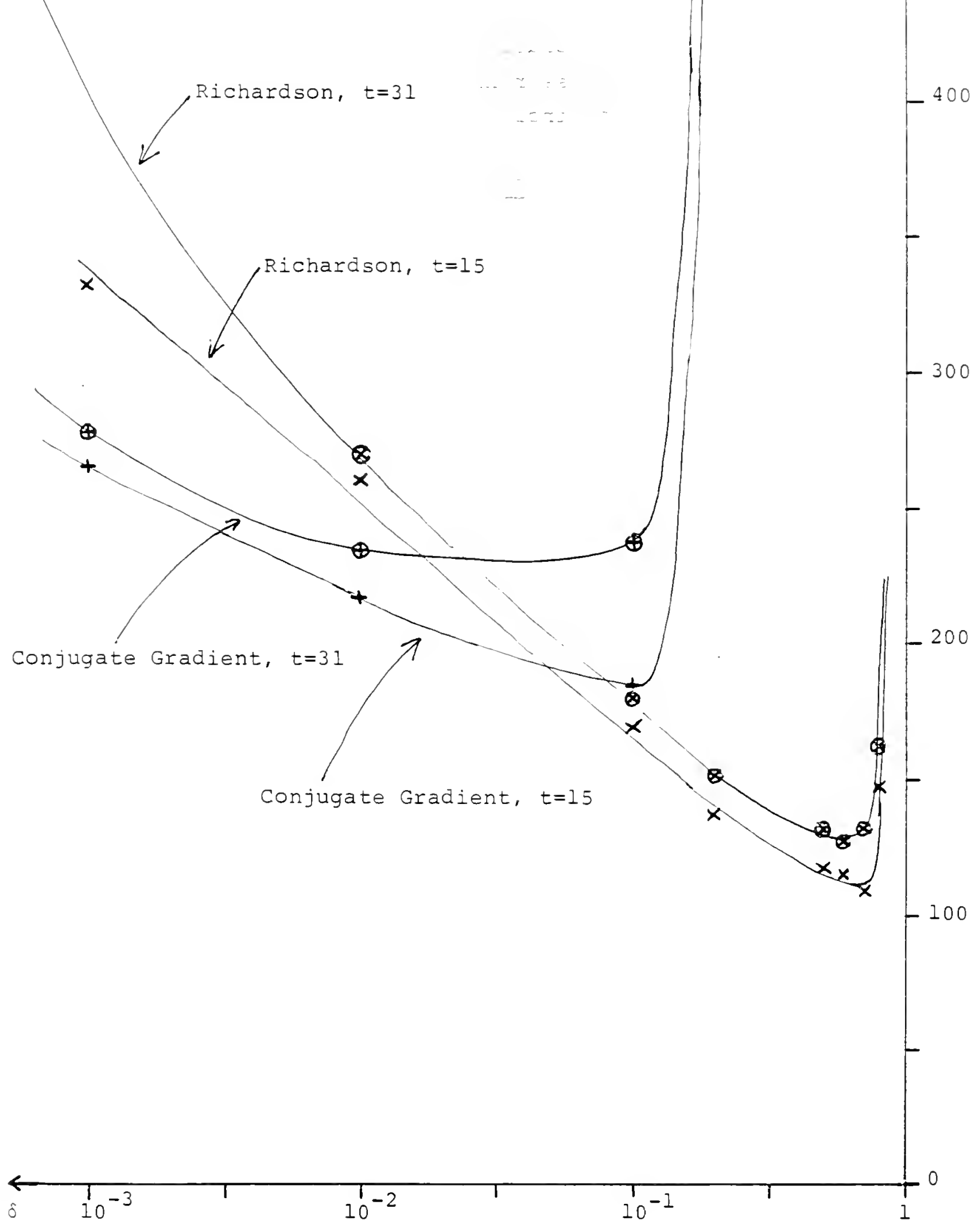


conjugate gradient method when the inner iterations are carried out quite accurately, but more efficient when a near optimal $\delta$ is used.

The numerical results were obtained using a VAX-1I/780 at the Courant Mathematics and Computing Laboratory. Double precision arithmetic, i.e. approximately 16 decimal digits of accuracy, was used. More extensive experiments have been made, but they will be reported in a subsequent paper.

\section{Acknowledgements.}

The authors would like to thank olof Widlund, Don Rose and Nancy Nichols for several helpful conversations and suggestions.

\section{References}

Concus, P. and G.H. Golub (1976). A generalized conjugate gradient method for nonsymmetric systems of equations, Computer science Dept. Report STAN-CS-76-646, Stanford University, Stanford, California.

Dembo, R.S., S.C. Eisenstat and T. Steihaug (1980). Inexact Newton methods, School of Organization and Management Report (Series \#47), Yale University, New Haven, Connecticut.

Golub, G.H. (1962). Bounds for the round-off errors in the Richardson second-order method, BIT, $\mathrm{pP} .212-223$.

Golub, G.H. and R.S. Varga (1961). Chebyshev semi-iterative methods, successive overrelaxation iterative methods, and second order Richardson iterative methods, Parts I and II, Numer. Math. 3, pp. $147-168$.

Gunn, J.E. (1964). The numerical solution of $\nabla \cdot a \nabla u=f$ by a semi-explicit alternating-direction iterative technique, Numer. Math. 6. pp. $181-184$.

Manteuffel, T.A. (1977). The Tchebyshev iteration for nonsymmetric linear systems, Numer. Math. 28, pp. 307-327.

Nichols, N.K. (1973). On the convergence of two-stage iterative processes for solving linear equations, SIAM J. Numer. Anal. 10, pp. $460-469$.

Nicolaides, R.A. (1975). On the local convergence of certain two step iterative procedures, Numer. Math. 24, pp. 95-10I.

Pereyra, V. (1967). Accelerating the convergence of discretization algorithms, SIAM J. Numer. Anal.4, pp. 508-533.

Varga, R.S. (1962). Matrix Iterative Analysis, Prentice-Hall, Englewood Cliffs, New Jersey. 
Widlund, 0 . (1978). A Lanczos method for a class of nonsymmetric systems of linear equations, SIAM J. Numer. Anal. 15, pp. 801-812.

Young, D.M. (1971). Iterative Solution of Large Linear systems, Academic Press, New York and London. 
Comp. Sci. Dept.

$\mathrm{TR}-38$

Golub

Convergence on a two-

stage Richardson it- $c .1$

NYU

CRMB 8 Goliub

Dept.

convergence of a two-

stage Richardson ...

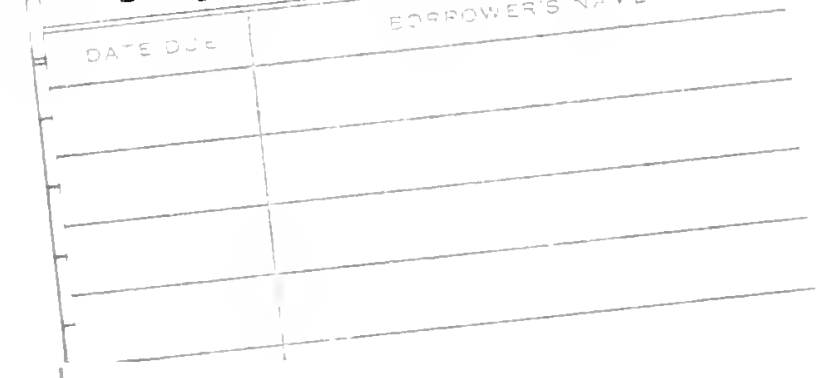

N.Y.U. Courant Institute of

Mathematical Sciences

251 Mercer St.

New York, N. Y. 10012

This book may be kept

FOURTEEN DAYS

A fine will be cbarged for each day the book is kept overtime.

\begin{tabular}{l|l|l|l}
\hline & & & \\
\hline & & & \\
\hline & & & \\
\hline & & & \\
\hline & & & \\
\hline & & & \\
\hline & & & \\
\hline & & & \\
\hline & & & \\
\hline & & & \\
\hline & & & \\
\hline & & & \\
\hline & & & \\
\hline GAYLORD 142 & & & FRINEO IN USA \\
\hline
\end{tabular}


